\title{
Nonexistence of almost Moore digraphs of diameter four
}

\author{
J. Conde*, J. Gimbert* \\ Dept. de Matemàtica, Universitat de Lleida \\ Jaume II, 69, 25001 Lleida, Spain \\ \{jconde, joangim\}@matematica.udl.cat \\ J. González ${ }^{\dagger}$ \\ Dept. de Matemàtica Aplicada IV, Universitat Politècnica de Catalunya \\ Víctor Balaguer s/n, 08800 Vilanova i Geltrú, Spain \\ josepg@ma4.upc.edu \\ J.M. Miret ${ }^{\ddagger}$, R. Moreno ${ }^{\ddagger}$ \\ Dept. de Matemàtica, Universitat de Lleida \\ Jaume II, 69, 25001 Lleida, Spain \\ \{miret, ramiro\}@matematica.udl.cat
}

Submitted: Jul 20, 2013; Accepted: Mar 25, 2013; Published: Mar 31, 2013

Mathematics Subject Classifications: 05C20, 05C50, 11R18

\begin{abstract}
Regular digraphs of degree $d>1$, diameter $k>1$ and order $N(d, k)=d+\cdots+d^{k}$ will be called almost Moore $(d, k)$-digraphs. So far, the problem of their existence has only been solved when $d=2,3$ or $k=2,3$. In this paper we prove that almost Moore digraphs of diameter 4 do not exist for any degree $d$.
\end{abstract}

Keywords: Almost Moore digraph, characteristic polynomial, cyclotomic polynomial.

\section{Introduction}

The degree/diameter problem finds, given two natural numbers $d$ and $k$, the largest possible number of vertices in a [directed] graph with maximum [out-]degree $d$ and diameter $k$ (for

*Partially supported by DGI Grant TIN2010-18978

†Partially supported by DGI Grant MTM2009-13060-C02-02

${ }^{\ddagger}$ Partially supported by DGI Grant MTM2010-21580-C02-01 
a survey of it see [12]). In the directed case, W.G. Bridges and S. Toueg in [4] proved that this number of vertices is less than the Moore bound, $M(d, k)=1+d+\cdots+d^{k}$, unless $d=1$ or $k=1$. Then, the question of finding for which values of $d>1$ and $k>1$ there exist digraphs of order

$$
N(d, k)=M(d, k)-1
$$

becomes an interesting problem. In this case, any extremal digraph turns out to be $d$ regular (see [10]). From now on, regular digraphs of degree $d>1$, diameter $k>1$ and order $N(d, k)$ will be called almost Moore $(d, k)$-digraphs (or $(d, k)$-digraphs for short).

The problem of the existence of almost Moore $(d, k)$-digraphs has been solved when $d=2,3$ or $k=2,3$. M. Miller and I. Fris [11] proved that the $(2, k)$-digraphs do not exist for values of $k>2$ and Baskoro et al. [3] established the nonexistence of $(3, k)$-digraphs unless $k=2$. On the other hand, Fiol et al. [6] showed that the $(d, 2)$-digraphs do exist for any degree. Their classification was completed by J. Gimbert in [8]. Moreover, J. Conde et al. [5] proved the nonexistence of $(d, 3)$-digraphs.

In this paper we prove that almost Moore digraphs of diameter four do not exist for any degree. The paper is organized as follows: Section 2 is devoted to determine the characteristic polynomial of a $(d, 4)$-digraph in terms of the polynomials $F_{n, 4}(x)=$ $\Phi_{n}\left(1+x+x^{2}+x^{3}+x^{4}\right)$, being $\Phi_{n}(x)$ the $n$th cyclotomic polynomial and $2 \leqslant n \leqslant N(d, 4)$. In Section 3, assuming the cyclotomic conjecture (see [7]) for $k=4$, which says that $F_{n, 4}(x)$ is irreducible unless $n=3,6$, we prove the nonexistence of $(d, 4)$-digraphs for $d \geqslant 2$. Finally, in Section 4 we show the conjecture for $k=4$.

\section{On the characteristic polynomial of a $(d, 4)$-digraph}

Given a $(d, k)$-digraph $G$, its adjacency matrix $A$ fulfills the equation

$$
I+A+\cdots+A^{k}=J+P,
$$

where $J$ denotes the all-one matrix and $P=\left(p_{i j}\right)$ is the $(0,1)$-matrix associated with a distinguished permutation $r$ of the set of vertices $V(G)=\{1, \ldots, N\}$; that is to say, $p_{i j}=1$ iff $r(i)=j$ (see [1]).

Notice that $r$ has a cycle structure which corresponds to its unique decomposition in disjoint cycles. The number of permutation cycles of $G$ of each length $n \leqslant N$ will be denoted by $m_{n}$ and the vector $\left(m_{1}, \ldots, m_{N}\right)$ will be referred to as the permutation cycle structure of $G$.

The factorization of $\operatorname{det}(x I-(J+P))$ in $\mathbb{Q}[x]$ in terms of the cyclotomic polynomials $\Phi_{i}(x)$ is given by (see $\left.[2,5]\right)$

$$
\operatorname{det}(x I-(J+P))=(x-(N+1))(x-1)^{m(1)-1} \prod_{n=2}^{N} \Phi_{n}(x)^{m(n)},
$$

where $m(n)=\sum_{n \mid i} m_{i}$ represents the total number of permutation cycles of order multiple of $n$. 
From Equations (1) and (2), the problem of the factorization in $\mathbb{Q}[x]$ of the characteristic polynomial of $G, \phi(G, x)=\operatorname{det}(x I-A)$, was connected by J. Gimbert in [7] with the study of the irreducibility in $\mathbb{Q}[x]$ of the polynomials

$$
F_{n, k}(x)=\Phi_{n}\left(1+x+\cdots+x^{k}\right) .
$$

The idea is that, when such polynomials are irreducible, they appear as factors of the characteristic polynomial of $G$.

Proposition 1. Let $\left(m_{1}, \ldots, m_{N}\right)$ be the permutation cycle structure of a $(d, k)$-digraph $G$ and $2 \leqslant n \leqslant N$. If $F_{n, k}(x)$ is an irreducible polynomial in $\mathbb{Q}[x]$, then it is a factor of $\phi(G, x)$ and its multiplicity is $m(n) / k$.

This result was proved in [7]. Moreover, it was proved that $F_{2, k}(x)=2+x+\cdots+x^{k}$ is irreducible in $\mathbb{Q}[x]$, for any positive integer $k$. On the other hand, it was shown that for each $n>2$ there are infinitely many values of $k$ for which $F_{n, k}(x)$ is reducible in $\mathbb{Q}[x]$. More precisely,

Lemma 2. Let $n>2$ and $k>1$ be integers. Then, the following statements hold.

(i) If $n$ is odd and $k \equiv-2(\bmod 2 n)$, then $\Phi_{2 n}(x)$ divides $F_{n, k}(x)$.

(ii) If $n \equiv 0(\bmod 4)$ and $k \equiv-2(\bmod n)$, then $\Phi_{n}(x)$ divides $F_{n, k}(x)$.

(iii) If $n \equiv 2(\bmod 4)$ and $k \equiv-2\left(\bmod \frac{n}{2}\right)$, then $\Phi_{\frac{n}{2}}(x)$ divides $F_{n, k}(x)$.

On the other hand, in [7] it was conjectured that $F_{n, k}(x)$ is irreducible in $\mathbb{Q}[x]$ if $n$ and $k$ do no satisfy any of the conditions of Lemma 2 .

Conjecture 3. Let $n>2$ and $k>1$ be integers. One has that

(i) If $k$ is even, then $F_{n, k}(x)$ is reducible in $\mathbb{Q}[x]$ if and only if $n \mid(k+2)$, in which case $F_{n, k}(x)$ has just two factors.

(ii) If $k$ is odd, then $F_{n, k}(x)$ is reducible in $\mathbb{Q}[x]$ if and only if $n$ is even and $n \mid 2(k+2)$, in which case $F_{n, k}(x)$ has just two factors.

We will refer to this conjecture as the cyclotomic conjecture. The case $k=2$ was proved by H.W. Lenstra Jr. and B. Poonen [9] and, recently, the authors proved the case $k=3$ in [5].

The remainder of this section is devoted to finding the conditions in order to obtain a factorization of the characteristic polynomial of a $(d, 4)$-digraph $G$ in terms of $F_{n, 4}(x)$. Thus, let $G$ be a $(d, 4)$-digraph of degree $d>3$ and let $\left(m_{1}, \ldots, m_{N}\right)$ be its permutation cycle structure, where $N=d+d^{2}+d^{3}+d^{4}$.

We will assume the cyclotomic conjecture is true for $k=4$, that is $F_{n, 4}(x)$ is irreducible in $\mathbb{Q}[x]$ except $n=3,6$, which will be proven in the last section. From now on, we will write $F_{n}(x)$ instead of $F_{n, 4}(x)$. 
Then, by applying Proposition 1 we have that

$$
\prod_{\substack{2 \leqslant n \leqslant N \\ n \neq 3,6}}\left(F_{n}(x)\right)^{\frac{m(n)}{4}} \text { is a factor of } \phi(G, x) .
$$

The remaining factors of $\phi(G, x)$ are derived as follows:

- Since $G$ is $d$-regular and strongly connected, $\phi(G, x)$ has the linear factor $x-d$ with multiplicity 1 ;

- Taking into account that $x-1$ is a factor of $\operatorname{det}(x I-(J+P))$ with multiplicity $m(1)-1$ and since

$$
F_{1}(x)=(x+1)\left(x^{2}+1\right) x
$$

we have that $x+1, x^{2}+1$ and $x$ are factors of $\phi(G, x)$ with multiplicities $a_{1}, a_{2}$ and $a_{3}$, respectively, where $a_{1}+2 a_{2}+a_{3}=m(1)-1$;

- Since $\Phi_{3}(x)=x^{2}+x+1$ is a factor of $\operatorname{det}(x I-(J+P))$ with multiplicity $m(3)$ and taking into account the factorization of $F_{3}(x)$ in $\mathbb{Q}[x]$,

$$
F_{3}(x)=\left(x^{2}-x+1\right)\left(x^{6}+3 x^{5}+5 x^{4}+6 x^{3}+7 x^{2}+6 x+3\right),
$$

we have that $\Phi_{6}(x)=x^{2}-x+1$ and $F_{3}(x) / \Phi_{6}(x)$ are factors of $\phi(G, x)$ with multiplicities $b_{1}$ and $b_{2}$, respectively, where $2 b_{1}+6 b_{2}=2 m(6)$; that is, $b_{1}=m(3)-$ $3 b_{2}$. Analogously, since the factorization of $F_{6}(x)$ in $\mathbb{Q}[x]$ is

$$
F_{6}(x)=\left(x^{2}+x+1\right)\left(x^{6}+x^{5}+x^{4}+2 x^{3}+x^{2}+1\right),
$$

we have that $\Phi_{3}(x)$ and $F_{6}(x) / \Phi_{3}(x)$ are factors of $\phi(G, x)$ with multiplicities $c_{1}$ and $c_{2}$, respectively, where $c_{1}=m(6)-3 c_{2}$.

As a result, the characteristic polynomial of $G$ is

$$
\begin{aligned}
\phi(G, x)= & (x-d)(x+1)^{a_{1}}\left(x^{2}+1\right)^{a_{2}} x^{a_{3}} \Phi_{6}(x)^{b_{1}}\left(F_{3}(x) / \Phi_{6}(x)\right)^{b_{2}} \\
& \times \Phi_{3}(x)^{c_{1}}\left(F_{6}(x) / \Phi_{3}(x)\right)^{c_{2}} \prod_{\substack{2 \leqslant n \leqslant N \\
n \neq 3,6}}\left(F_{n}(x)\right)^{\frac{m(n)}{4}} .
\end{aligned}
$$

\section{On the nonexistence of $(d, 4)$-digraphs}

In this section, we will derive the nonexistence of a $(d, 4)$-digraph from the irreducibility of the polynomials $F_{n}(x)$ which appear in the factorization of its characteristic polynomial and from the behaviour of the first three powers of its adjacency matrix.

Theorem 4. Assuming that the cyclotomic conjecture is true for $k=4$, there is no almost Moore digraph of diameter four. 
Proof. Let $G$ be a $(d, 4)$-digraph with adjacency matrix $A$. We compute the graph spectral invariants $\operatorname{Tr} A^{\ell}(\ell=1,2,3)$ in terms of the sum of the $\ell$ th powers of the roots of each factor of $\phi(G, x)$.

Given a monic polynomial of degree $n \geqslant 1, a(x)=x^{n}+\sum_{i=1}^{n} a_{n-i} x^{n-i}$, and given an integer $\ell \geqslant 1$, we define $S_{\ell}(a(x))$ to be the sum of the $\ell$ th powers of all the roots of $a(x)$. Using Newton's formulas [14], which express $S_{\ell}(a(x))$ in terms of the coefficients of $a(x)$, we have

$$
\begin{aligned}
& S_{1}(a(x))=-a_{n-1}, \\
& S_{2}(a(x))=a_{n-1}^{2}-2 a_{n-2}, \\
& S_{3}(a(x))=-a_{n-1}^{3}+3 a_{n-1} a_{n-2}-3 a_{n-3} .
\end{aligned}
$$

Since $S_{\ell}(a(x) b(x))=S_{\ell}(a(x)) S_{\ell}(b(x))$, for all pairs of polynomials, and taking into account that

$$
F_{n}(x)=\Phi_{n}\left(1+x+x^{2}+x^{3}+x^{4}\right)=\left(1+x+x^{2}+x^{3}+x^{4}\right)^{\varphi(n)}+O\left(x^{4 \varphi(n)-4}\right),
$$

where $\varphi(n)$ stands for Euler's function, we obtain

$$
S_{\ell}\left(F_{n}(x)\right)=\varphi(n) S_{\ell}\left(x^{4}+x^{3}+x^{2}+x+1\right)=-\varphi(n), \quad \ell=1,2,3 .
$$

Besides, it can be easily checked that

\begin{tabular}{c|r|r|r} 
& $S_{1}$ & $S_{2}$ & $S_{3}$ \\
\hline$x+1$ & -1 & 1 & -1 \\
$x^{2}+1$ & 0 & -2 & 0 \\
$\Phi_{6}(x)$ & 1 & -1 & -2 \\
$\Phi_{3}(x)$ & -1 & -1 & 2
\end{tabular}

Now, for each $\ell=1,2,3$ we can express the trace of the $\ell$ th power of the adjacency matrix $A$ of $G$ in terms of the sums $S_{\ell}$ of all factors of $\phi(G, x)$. Thus,

$$
\begin{aligned}
\operatorname{Tr} A & =d-a_{1}+b_{1}-3 b_{2}-c_{1}-c_{2}-\frac{1}{4} T, \\
\operatorname{Tr} A^{2} & =d^{2}+a_{1}-2 a_{2}-b_{1}-b_{2}-c_{1}-c_{2}-\frac{1}{4} T, \\
\operatorname{Tr} A^{3} & =d^{3}-a_{1}-2 b_{1}+2 c_{1}-4 c_{2}-\frac{1}{4} T,
\end{aligned}
$$

where $T=\sum_{\substack{2 \leqslant n \leqslant N \\ n \neq 3,6}} m(n) \varphi(n)$. From the identity $\sum_{n=1}^{N} m(n) \varphi(n)=N$ (see [7]),

$$
T=N-m(1)-2 m(3)-2 m(6) .
$$

So, taking into account that $b_{1}=m(3)-3 b_{2}$ and $c_{1}=m(6)-3 c_{2}$,

$$
\begin{aligned}
& \operatorname{Tr} A=d-\frac{1}{4} N+\frac{1}{4} m(1)+\frac{3}{2} m(3)-\frac{1}{2} m(6)-a_{1}-6 b_{2}+2 c_{2}, \\
& \operatorname{Tr} A^{2}=d^{2}-\frac{1}{4} N+\frac{1}{4} m(1)-\frac{1}{2} m(3)-\frac{1}{2} m(6)+a_{1}-2 a_{2}+2 b_{2}+2 c_{2}, \\
& \operatorname{Tr} A^{3}=d^{3}-\frac{1}{4} N+\frac{1}{4} m(1)-\frac{3}{2} m(3)+\frac{5}{2} m(6)-a_{1}+6 b_{2}-10 c_{2} .
\end{aligned}
$$


Since $G$ has no cycles of length $\leqslant 3$, we know that $\operatorname{Tr} A^{\ell}=0(\ell=1,2,3)$. As a consequence,

$$
\begin{aligned}
4 a_{1} & +24 b_{2}-8 c_{2}=4 d-N+m(1)+6 m(3)-2 m(6) \\
-4 a_{1}+8 a_{2}-8 b_{2}-8 c_{2} & =4 d^{2}-N+m(1)-2 m(3)-2 m(6) \\
4 a_{1} & -24 b_{2}+40 c_{2}=4 d^{3}-N+m(1)-6 m(3)+10 m(6)
\end{aligned}
$$

Applying Gauss reduction method to the previous linear system, it follows that

$$
\begin{aligned}
8 a_{2}+16 b_{2}-16 c_{2} & =4 d^{2}+4 d-2 N+2 m(1)+4 m(3)-4 m(6), \\
-48 b_{2}+48 c_{2} & =4 d^{3}-4 d-12 m(3)+12 m(6) .
\end{aligned}
$$

Taking into account that $N=d^{4}+d^{3}+d^{2}+d$, from (5) and (6) we derive that

$$
24 a_{2}=4 d^{3}+12 d^{2}+8 d+6 m(1)-6 N .
$$

Notice that $m(1)=\sum_{n=1}^{N} m_{n}$ takes its maximum value when all permutation cycles are short as possible. Moreover, the number of selfrepeats $m_{1}$ of a $(d, k)$-digraph is either 0 or $k$, if $k \geqslant 3$ (see [1]). So, $m(1) \leqslant 4+\frac{N-4}{2}$ and, consequently,

$$
24 a_{2} \leqslant 4 d^{3}+12 d^{2}+8 d+12-3 N=-3 d^{4}+d^{3}+9 d^{2}+5 d+12 .
$$

Hence, if $d>3$ then $a_{2}<0$, which is impossible since $a_{2}$ is a nonnegative integer.

\section{The cyclotomic conjecture for $k=4$}

This section is devoted to proving the cyclotomic conjecture in the case $k=4$, that is, we show that the polynomial $F_{n}(x)=\Phi_{n}\left(1+x+x^{2}+x^{3}+x^{4}\right)$ is irreducible in $\mathbb{Q}[x]$, when $n>1$ and $n \neq 3,6$.

As a first step, we show that the condition of being $F_{n}(x)$ reducible in $\mathbb{Q}[x]$ implies a divisibility relation by a cyclotomic polynomial. In order to prove this, let us suppose that $F_{n}(x)$ is reducible in $\mathbb{Q}[x]$ and let us consider a root $\varepsilon$ of $F_{n}(x)$. Denoting

$$
p_{1}(x, z)=1-z+x+x^{2}+x^{3}+x^{4}
$$

and taking a suitable primitive $n$th root of unity $\zeta_{n}$, we get

$$
p_{1}\left(\varepsilon, \zeta_{n}\right)=0
$$

Using properties about the degrees of the algebraic extensions

$$
\mathbb{Q} \subseteq \mathbb{Q}\left(\zeta_{n}\right) \subseteq \mathbb{Q}(\varepsilon)
$$

we derive that $F_{n}(x)$ has an irreducible factor in $\mathbb{Q}[x]$ of degree $\varphi(n)$ or $2 \varphi(n)$. We can assume that $\varepsilon$ is a root of such a factor. In particular, $\varepsilon$ is an algebraic integer and $\left[\mathbb{Q}(\varepsilon): \mathbb{Q}\left(\zeta_{n}\right)\right]$ is either 1 or 2 . 
If $\left[\mathbb{Q}(\varepsilon): \mathbb{Q}\left(\zeta_{n}\right)\right]=1$, we consider the element $\bar{\varepsilon} / \varepsilon \in \mathbb{Q}(\varepsilon, \bar{\varepsilon})$, where ${ }^{-}$denotes the complex conjugation. By using arguments given in [5] we obtain that $\bar{\varepsilon} / \varepsilon$ is a root of unity and hence the same procedure given for diameter 3 to state the irreducibility of $F_{n}(x)$ follows.

Now, assume that $\left[\mathbb{Q}(\varepsilon): \mathbb{Q}\left(\zeta_{n}\right)\right]=2$ for all $\varepsilon$ such that $p_{1}\left(\varepsilon, \zeta_{n}\right)=0$. We denote by $\varepsilon^{\prime}$ the conjugate root of $\varepsilon$ over $\mathbb{Q}\left(\zeta_{n}\right)$, that is to say, the polynomial $p_{1}\left(x, \zeta_{n}\right) /\left((x-\varepsilon)\left(x-\varepsilon^{\prime}\right)\right)$ is irreducible in $\mathbb{Q}\left(\zeta_{n}\right)[x]$. Changing the root of $p_{1}\left(x, \zeta_{n}\right)$ if necessary, we can assume that $\varepsilon \varepsilon^{\prime}$ is not real. Since $\varepsilon$ is an algebraic integer and $1-\zeta_{n}$ is a unity or a prime element of $\mathbb{Z}\left[\zeta_{n}\right], \varepsilon \varepsilon^{\prime}$ is also a unity or a prime element of $\mathbb{Z}\left[\zeta_{n}\right]$. Therefore,

$$
\alpha=\frac{\overline{\varepsilon \varepsilon^{\prime}}}{\varepsilon \varepsilon^{\prime}} \in \mathbb{Z}\left[\zeta_{n}\right]
$$

is a unity of $\mathbb{Z}\left[\zeta_{n}\right]$ whose conjugates have absolute value 1 . Hence, $\alpha \neq 1$ is a root of unity of order $2 n$ [15, Lemma 1.6]. Notice that if $n$ is even, $\alpha$ is a root of unity of order $n$.

Now, we search for a polynomial relation between $\zeta_{n}$ and $\alpha=\beta \beta^{\prime}$, where $\beta=\bar{\varepsilon} / \varepsilon$ and $\beta^{\prime}=\bar{\varepsilon}^{\prime} / \varepsilon^{\prime}$. In order to find such an expression we give first a relation between $\zeta_{n}$ and $\beta$. We use the following identities:

$$
\begin{aligned}
1+\varepsilon+\varepsilon^{2}+\varepsilon^{3}+\varepsilon^{4} & =\zeta_{n}, \\
\bar{\varepsilon} & =\beta \varepsilon .
\end{aligned}
$$

From them, and taking into account that $\overline{\zeta_{n}}=1 / \zeta_{n}$, it can be seen that $p_{2}\left(\varepsilon, \beta, \zeta_{n}\right)=0$ where

$$
p_{2}(x, y, z)=1-z-x y z-x^{2} y^{2} z-x^{3} y^{3} z-x^{4} y^{4} z .
$$

Similarly, $p_{2}\left(\varepsilon^{\prime}, \beta^{\prime}, \zeta_{n}\right)=0$. Notice as well that $p_{3}\left(\alpha, \beta, \beta^{\prime}\right)=0$ where

$$
p_{3}\left(y, y^{\prime}, w\right)=w-y y^{\prime} .
$$

Therefore, the relation between $\zeta_{n}$ and $\alpha$ we are looking for is $R\left(\zeta_{n}, \alpha\right)=0$, where

$$
\begin{aligned}
R_{1}(y, z) & =\operatorname{Res}\left(p_{1}(x, z), p_{2}(x, y, z), x\right), \\
R_{2}\left(y^{\prime}, z, w\right) & =\operatorname{Res}\left(R_{1}(y, z), p_{3}\left(y, y^{\prime}, w\right), y\right), \\
R(z, w) & =\operatorname{Res}\left(R_{1}\left(y^{\prime}, z\right), R_{2}\left(y^{\prime}, z, w\right), y^{\prime}\right) .
\end{aligned}
$$

This polynomial factorizes as follows

$$
R(z, w)=(z-1)^{50} q_{1}(z, w) q_{2}^{2}(z, w) q_{3}^{2}(z, w) q_{4}^{4}(z, w)
$$

where $q_{1}(z, w)$ has degree 14 in $z$ and 16 in $w, q_{2}(z, w)$ and $q_{3}(z, w)$ have degree 21 in $z$ and 24 in $w$, and $q_{4}(z, w)$ has degree 27 in $z$ and 36 in $w$.

Proposition 5. Let $n>2$ be an integer and $F_{n}(x)=\Phi_{n}\left(1+x+x^{2}+x^{3}+x^{4}\right)$. If $F_{n}(x)$ is reducible in $\mathbb{Q}[x]$ then: 
- If $n$ is even, then there exists an integer $k, 1 \leqslant k<n$, such that $\Phi_{n}(x)$ divides one of the polynomials $q_{i}\left(x, x^{k}\right), i \in\{1,2,3,4\}$, given in (12).

- If $n$ is odd, then there exists an integer $k, 1 \leqslant k<n$, such that $\Phi_{n}(x)$ divides one of the polynomials $q_{i}\left(x, x^{k}\right)$ or $q_{i}\left(x,-x^{k}\right), i \in\{1,2,3,4\}$, given in (12).

Proof. Since the cyclotomic polynomial $\Phi_{n}(x)$ is irreducible in $\mathbb{Q}[x]$ and it does not divide $x-1$, then when $n$ is even it must divide at least one of the polynomials $q_{i}\left(x, x^{k}\right)$, $i \in\{1,2,3,4\}, 1 \leqslant k<n$. When $n$ is odd, $\alpha$ or $-\alpha$ is a root of unity of order $n$. Hence, $\Phi_{n}(x)$ must divide $q_{i}\left(x, x^{k}\right)$ or $q_{i}\left(x,-x^{k}\right), i \in\{1,2,3,4\}$.

Our main goal is to show that $F_{n}(x)$ is irreducible in $\mathbb{Q}[x]$, for $n>1$ and $n \neq 3,6$. It is enough to prove that $\Phi_{n}(x)$ does not divide, for $i \in\{1,2,3,4\}$, any of the polynomials $q_{i}\left(x, x^{k}\right), 1 \leqslant k<n$, when $n$ is even and it does not divide any of the polynomials $q_{i}\left(x, x^{k}\right)$ or $q_{i}\left(x,-x^{k}\right), 1 \leqslant k<n$, when $n$ is odd. This is equivalent to proving that $\Phi_{2 n}(x)$ does not divide any of the polynomials $q_{i}\left(x^{2}, x^{\ell}\right), 1 \leqslant \ell<2 n$.

Theorem 6. The polynomial $F_{n}(x)$ is irreducible in $\mathbb{Q}[x]$ for $n>1$, unless $n=3,6$.

Proof. If $F_{n}(x)$ is reducible, then taking into account Proposition 5 there exist polynomials $q_{i}\left(x^{2}, x^{\ell}\right), i \in\{1,2,3,4\}$, given by (12) such that the cyclotomic polynomial $\Phi_{2 n}(x)$ divides one of them. Now, we show that $\Phi_{2 n}(x)$ does not divide $q_{1}\left(x^{2}, x^{\ell}\right)$. To see this, from part (i) of Lemma 3 in [5] (see also [13]), we know that

$$
\Phi_{2 n}(x) \equiv \Phi_{r}(x)^{\varphi\left(p^{e}\right)} \quad(\bmod p \mathbb{Z}[x]),
$$

where $p$ is a prime number dividing $2 n$ with $2 n=p^{e} r$ and $(p, r)=1$. Consequently

$$
\Phi_{r}(x)^{\varphi\left(p^{e}\right)-1} \mid \operatorname{gcd}\left(q_{1}\left(x^{2}, x^{\ell}\right), x q_{1}^{\prime}\left(x^{2}, x^{\ell}\right)\right) \quad(\bmod p \mathbb{Z}[x]) .
$$

Now, we consider the polynomial

$$
A_{1}(z, w)=2 z \frac{\partial}{\partial z} q_{1}(z, w)+\ell w \frac{\partial}{\partial w} q_{1}(z, w) \in \mathbb{Z}[z, w]
$$

that is $A_{1}\left(x^{2}, x^{\ell}\right)=x q_{1}^{\prime}\left(x^{2}, x^{\ell}\right)$. Therefore

$$
\Phi_{r}(x)^{\varphi\left(p^{e}\right)-1} \mid P_{1}(x) \quad(\bmod p \mathbb{Z}[x]),
$$

where $P_{1}(x)$ is the following resultant

$$
P_{1}(x)=\operatorname{Res}\left(q_{1}\left(x^{2}, w\right), A_{1}\left(x^{2}, w\right), w\right) .
$$

It can be checked that

$$
P_{1}(x)=5^{4} x^{264} \Phi_{1}^{82}(x) \Phi_{2}^{82}(x) \Phi_{4}^{12}(x) \Phi_{3}^{6}(x) \Phi_{6}^{6}(x) \Phi_{12}^{6}(x) P_{1,0}^{2}(x) P_{1, \ell}(x),
$$

with $P_{1,0}(x)$ a polynomial of degree 36 and $P_{1, \ell}(x)$ a polynomial of degree at most 60 . 
Notice that for those integers $n$ which have a prime factor $p$ such that $P_{1}(x) \neq 0$ $(\bmod p \mathbb{Z}[x])$ for all $\ell(\bmod p)$, the degree of $P_{1}(x)(\bmod p \mathbb{Z}[x])$ provides us an upper bound $K$ for $\varphi(n)$. Hence, for those values of $n$ such that $\varphi(n)>K, F_{n}(x)$ is irreducible in $\mathbb{Q}[x]$, and for those $n$ with $\varphi(n) \leqslant K$, we can computationally check the irreducibility of $F_{n}(x)$ unless $n=3,6$.

The coefficients of $P_{1,0}(x)$ do not depend on $\ell$ and its gcd is one. Hence, this polynomial does not vanish for any prime $p$. The polynomial $P_{1, \ell}(x)$ is given by

$$
P_{1, \ell}(x)=\sum_{i=0}^{30} a_{i}(\ell) x^{2 i},
$$

where the coefficients $a_{i}(\ell)$ are polynomials on $\mathbb{Q}[\ell]$ of degree 16 given by the expressions

$$
\begin{aligned}
a_{0}(\ell)= & 2^{32} 5^{12}(\ell+1)^{16} \\
a_{1}(\ell)= & -2^{26} 5^{11}(\ell+1)^{12}\left(9353 \ell^{4}+37412 \ell^{3}+57248 \ell^{2}+39552 \ell+10368\right), \\
a_{2}(\ell)= & 2^{17} 5^{10}(\ell+1)^{8}\left(338813683 \ell^{8}+2710509464 \ell^{7}+9562778864 \ell^{6}\right. \\
& +19424004608 \ell^{5}+24833262080 \ell^{4}+20453500928 \ell^{3}+10593286144 \ell^{2} \\
& +3152707584 \ell+412581888), \\
& \vdots \\
a_{29}(\ell)= & -2^{26} 5^{11}(\ell+1)^{12}\left(9353 \ell^{4}+37412 \ell^{3}+57248 \ell^{2}+39552 \ell+10368\right), \\
a_{30}(\ell)= & 2^{32} 5^{12}(\ell+1)^{16} .
\end{aligned}
$$

From the first coefficient it turns out that the factors which can vanish $P_{1, \ell}(x)$ are 2,5 and those that divide $\ell+1$. The polynomials $a_{j}(\ell), j=4, \ldots, 26$, are not divisible by $\ell+1$. The greatest common divisor of the remaining divisions of these polynomials by $\ell+1$ in $\mathbb{Z}[x]$ is 1 . Thus, there are no primes dividing $\ell+1$ that vanish $P_{1, \ell}(x)$. For the prime $p=2$, the polynomial $P_{1, \ell}(x)$ only vanishes when $\ell$ is even. Concerning the prime $p=5$, the polynomial $P_{1, \ell}(x)$ only vanishes when $\ell \equiv 4(\bmod 5)$.

Now, if the factorization of $n$ has a prime factor $p$ different from 2 and 5 , by using (13) and taking into account the factorization of $P_{1}(x)(\bmod p \mathbb{Z}[x])$ given in (14), the degree of the maximum power $\Phi_{r}(x)$ that could divide $P_{1}(x)(\bmod p \mathbb{Z}[x])$ is bounded by $\operatorname{deg} P_{1}(x)-\operatorname{deg} x^{264}=368$. This is a bound for $\left(\varphi\left(p^{e}\right)-1\right) \varphi(r)$. Hence,

$$
\varphi(n) \leqslant \varphi(2 n)=\varphi\left(p^{e}\right) \varphi(r) \leqslant 368+\varphi(r) \leqslant 736 .
$$

For these integers $n$ which have a prime factor different from 2 and 5 and such that $\varphi(n)>736, F_{n}(x)$ is irreducible in $\mathbb{Q}[x]$. For those integers $n$ such that $\varphi(n) \leqslant 736$, it has been computationally checked that $F_{n}(x)$ is reducible in $\mathbb{Q}[x]$ only when $n=3$ and $n=6$. Therefore, the remaining cases to consider are $n=2^{e} 5^{d}$, with $e \geqslant 1$ or $d \geqslant 1$.

The previous method works as well taking $p=2$ in (13) when $\ell$ is odd. On the other hand, if $5 \mid n$ and $p=5$, then $P_{1}(x)=0(\bmod p \mathbb{Z}[x])$ but the following relation holds

$$
\Phi_{r}(x)^{\varphi\left(p^{e}\right)-2} \mid Q_{1}(x) \quad(\bmod p \mathbb{Z}[x]),
$$


where $Q_{1}(x)$ is the resultant

$$
Q_{1}(x)=\operatorname{Res}\left(q_{1}\left(x^{2}, w\right), B_{1}\left(x^{2}, w\right), w\right)
$$

being

$$
B_{1}(z, w)=2 z \frac{\partial}{\partial z} A_{1}(z, w)+k w \frac{\partial}{\partial w} A_{1}(z, w) .
$$

Since we must consider the cases $n=2^{e} 5^{d}$, we can apply (15) with $p=5$ and we proceed in the same way as in (13). Nevertheless, the polynomial $Q_{1}(x)(\bmod 5 \mathbb{Z}[x])$ is identically zero only for $\ell \equiv 4(\bmod 5)$. Thus, taking into account these remarks, the cases we must study have been reduced to the following:

i) $n=2^{e} 5^{d}$, with $e \geqslant 0, d>0, \ell$ even and $\ell \equiv 4(\bmod 5)$,

ii) $n=2^{e}$, with $e \geqslant 1$, and $\ell$ even.

i) We shall prove that $\Phi_{2 n}(x)(\bmod 5 \mathbb{Z}[x])$ does not divide $q_{1}\left(x^{2}, x^{\ell}\right)(\bmod 5 \mathbb{Z}[x])$, for $\ell$ even and $\ell \equiv 4(\bmod 5)$. It is known that $\Phi_{2 n}(x)=\Phi_{2^{e+1}}(x)^{4 \cdot 5^{d-1}}(\bmod 5 \mathbb{Z}[x])$, where

$$
\Phi_{2^{m}}(x) \quad(\bmod 5 \mathbb{Z}[x])= \begin{cases}x+4 & \text { if } m=0 \\ x+1 & \text { if } m=1 \\ \left(x^{2^{m-2}}+2\right)\left(x^{2^{m-2}}+3\right) & \text { if } m \geqslant 2\end{cases}
$$

We have that

$$
q_{1}(z, w)=q_{1,1}(z, w)^{2} q_{1,2}(z, w) q_{1,3}(z, w) q_{1,4}(z, w) \quad(\bmod 5 \mathbb{Z}[z, w])
$$

where

$$
\begin{aligned}
q_{1,1}(z, w)= & w^{2} z-1, \\
q_{1,2}(z, w)= & w^{4} z^{4}-2 w^{4} z^{3}+w^{4} z^{2}+w^{3} z^{2}-2 w^{2} z^{3}+w^{2} z^{2}-2 w^{2} z+w z^{2}+z^{2}-2 z+1, \\
q_{1,3}(z, w)= & w^{4} z^{4}-2 w^{4} z^{3}+w^{4} z^{2}-2 w^{3} z^{3}-2 w^{3} z^{2}-2 w^{2} z^{3}+2 w^{2} z^{2}-2 w^{2} z-2 w z^{2} \\
& -2 w z+z^{2}-2 z+1 \\
q_{1,4}(z, w)= & w^{4} z^{4}-2 w^{4} z^{3}+w^{4} z^{2}-w^{3} z^{3}-2 w^{2} z^{3}+w^{2} z^{2}-2 w^{2} z-w z+z^{2}-2 z+1 .
\end{aligned}
$$

So, we will prove that $\Phi_{2^{e+1} 5^{d}}(x)(\bmod 5 \mathbb{Z}[x])$ does not divide $q_{1, i}\left(x^{2}, x^{\ell}\right)(\bmod 5 \mathbb{Z}[x])$, for any $i \in\{1,2,3,4\}$, when $e>0$ and $e=0$.

- Case $e>0$. First, we claim that

$$
\operatorname{gcd}\left(\Phi_{2^{e+1}}(x) \quad(\bmod 5 \mathbb{Z}[x]), q_{1,1}\left(x^{2}, x^{\ell}\right) \quad(\bmod 5 \mathbb{Z}[x])\right)=1 .
$$

Indeed, let $\gamma$ be a root of $\Phi_{2^{e+1}}(x)(\bmod 5 \mathbb{Z}[x])$, that is $\gamma^{2^{e-1}}$ is equal to 2 or 3 . Then, $\gamma^{2^{e+1}}$ is the smallest power of $\gamma$ equal to 1 . Therefore, if $\gamma$ is a root of $q_{1,1}\left(x^{2}, x^{\ell}\right)=x^{2(\ell+1)}-1$ then $2^{e+1} \mid 2(\ell+1)$, which contradicts that $\ell$ is even.

Assume $\Phi_{2^{e+1}}(x)(\bmod 5 \mathbb{Z}[x])$ divides $q_{1,2}\left(x^{2}, x^{\ell}\right) q_{1,3}\left(x^{2}, x^{\ell}\right) q_{1,4}\left(x^{2}, x^{\ell}\right)$. Then each irreducible divisor of $\Phi_{2^{e+1}}(x)(\bmod 5 \mathbb{Z}[x])$ is a divisor of some of the polynomials $q_{1, i}\left(x^{2}, x^{\ell}\right)$ 
$(\bmod 5 \mathbb{Z}[x]), i \in\{2,3,4\}$, with multiplicity greater than 1 . Then, for $i \in\{2,3,4\}$ we consider the resultant

$$
T_{1, i}(x)=\operatorname{Res}\left(q_{1, i}\left(x^{2}, w\right), S_{1, i}\left(x^{2}, w\right), w\right),
$$

where

$$
S_{1, i}(z, w)=2 z \frac{\partial}{\partial z} q_{1, i}(z, w)+\ell w \frac{\partial}{\partial w} q_{1, i}(z, w) .
$$

When $\ell=4(\bmod 5)$, the polynomials $T_{1, i}(x)(\bmod 5 \mathbb{Z}[x])$ are as follows:

$$
\begin{aligned}
& T_{1,2}(x)=x^{20}(1+x)^{6}(2+x)^{2}(3+x)^{2}(4+x)^{6}\left(1+x+x^{2}\right)^{2}\left(1+4 x+x^{2}\right)^{2}, \\
& T_{1,3}(x)=x^{20}(1+x)^{4}(4+x)^{4}\left(4+2 x+x^{2}\right)^{4}\left(4+3 x+x^{2}\right)^{4}, \\
& T_{1,4}(x)=x^{20}(1+x)^{6}(2+x)^{2}(3+x)^{2}(4+x)^{6}\left(1+x+x^{2}\right)^{2}\left(1+4 x+x^{2}\right)^{2} .
\end{aligned}
$$

Therefore, $e$ must be 1 and $\Phi_{4}(x)^{7}(\bmod 5 \mathbb{Z}[x])$ is the greatest power of $\Phi_{4}(x)(\bmod 5 \mathbb{Z}[x])$ which could divide $q_{1,2}\left(x^{2}, x^{\ell}\right) q_{1,3}\left(x^{2}, x^{\ell}\right) q_{1,4}\left(x^{2}, x^{\ell}\right)$. Since

$$
\Phi_{2^{e+1} 5^{d}}(x)=\Phi_{2^{e+1}}(x)^{4 \cdot 5^{d-1}} \quad(\bmod 5 \mathbb{Z}[x]),
$$

for $d>1$ the polynomial $\Phi_{2^{e+1} 5^{d}}(x)(\bmod 5 \mathbb{Z}[x])$ does not divide $q_{1}\left(x^{2}, x^{\ell}\right)(\bmod 5 \mathbb{Z}[x])$. For $n=2 \cdot 5$ we can check that $F_{n}(x)$ is irreducible in $\mathbb{Q}[x]$.

- Case $e=0$. In this case $\Phi_{2 \cdot 5^{d}}(x)=(x+1)^{4 \cdot 5^{d-1}}(\bmod 5 \mathbb{Z}[x])$. Set $\ell+1=5^{k} m$ with $m$ odd and $\operatorname{gcd}(5, m)=1$. Since $\ell+1=0(\bmod 5)$ and $\ell+1 \leqslant 2 \cdot 5^{d}$, it is clear that $1 \leqslant k \leqslant d$. The polynomial $(x+1)^{2 \cdot 5^{k}}$ is the greatest power of $x+1$ which divides $q_{1,1}\left(x^{2}, x^{\ell}\right)^{2}=\left(x^{2(\ell+1)}-1\right)^{2}=\left(x^{m}-1\right)^{2 \cdot 5^{k}}\left(x^{m}+1\right)^{2 \cdot 5^{k}}(\bmod 5 \mathbb{Z}[x])$. From the following equalities

$$
\operatorname{Res}\left(q_{1,1}\left(x^{2}, w\right), q_{1, i}\left(x^{2}, w\right), w\right)=4 x^{10}(x+1)^{2}(4+x)^{2}, \quad 2 \leqslant i \leqslant 4,
$$

we get that $(x+1)^{2 \cdot 5^{k}+6}$ is the greatest power of $x+1$ dividing $q_{1}\left(x^{2}, x^{\ell}\right)$. Hence, $4 \cdot 5^{d-1} \leqslant$ $2 \cdot 5^{k}+6$ and, thus, $k=d$. So, $\ell+1$ must be either $5^{d}$ or $2 \cdot 5^{d}$. Since $\ell$ is even, $\ell=5^{d}-1$. Therefore, only for this value of $\ell$ the polynomial $\Phi_{2 \cdot 5^{d}}(x)$ can divide $q_{1}\left(x^{2}, x^{\ell}\right)$. Nevertheless, since the roots of $\Phi_{2 \cdot 5^{d}}(x)$ satisfy that $x^{5^{d}}=-1$, the polynomial $\Phi_{2 \cdot 5^{d}}(x)$ should divide

$$
q_{1}\left(x^{2},-1 / x\right)=25(-1+x)^{4}(1+x)^{6}\left(1-x+x^{2}\right),
$$

which leads to a contradiction.

ii) In this case $n=2^{e}$, with $e \geqslant 1$ and $\ell=2 k$. We shall prove that $\Phi_{2^{e}}(x)(\bmod 5 \mathbb{Z}[x])$ does not divide $q_{1}\left(x, x^{k}\right)(\bmod 5 \mathbb{Z}[x])$. With the same arguments used in the above case, we obtain that

$$
\operatorname{gcd}\left(\Phi_{2^{e}}(x)(\bmod 5 \mathbb{Z}[x]), q_{1,1}\left(x, x^{k}\right) \quad(\bmod 5 \mathbb{Z}[x])\right)=1
$$


Let $\gamma \in \mathbb{F}_{5^{2^{e-2}}}$ such that $\Phi_{2^{e}}(\gamma)=0$, where $\mathbb{F}_{5^{2^{e-2}}}$ is the finite field with $5^{e-2}$ elements. Since $\Phi_{2^{e}}(x)=\left(x^{2^{e-2}}+2\right)\left(x^{2^{e-2}}+3\right)$ is the decomposition in irreducible factors in $\mathbb{F}_{5}$, we know that $\mathbb{F}_{5^{2 e-2}}=\mathbb{F}_{5}(\gamma)$ and $\gamma^{2^{e-2}}=a$, where $a$ is either 2 or 3. Moreover,

$$
\operatorname{Tr}\left(\gamma^{m}\right)=\left\{\begin{array}{cl}
\varphi\left(2^{e}\right) / 2 & \text { if } \operatorname{gcd}\left(m, 2^{e}\right)=2^{e} \\
-\varphi\left(2^{e}\right) / 2 & \text { if } \operatorname{gcd}\left(m, 2^{e}\right)=2^{e-1} \\
\pm a \varphi\left(2^{e}\right) / 2 & \text { if } \operatorname{gcd}\left(m, 2^{e}\right)=2^{e-2} \\
0 & \text { otherwise }
\end{array}\right.
$$

where $\operatorname{Tr}$ denotes the trace $\operatorname{Tr}_{\mathbb{F}_{5^{2}}-2} / \mathbb{F}_{5}$ and the sign of $a$ depends on the class $\frac{m}{2^{e-2}}(\bmod 4)$. We can assume that $e>5$ and, thus, when $\operatorname{gcd}\left(m, 2^{e}\right) \mid 8$ we have $\operatorname{Tr}\left(\gamma^{m}\right) \stackrel{2}{=} 0$. If

$$
q_{1,4}\left(\gamma, \gamma^{\ell}\right)=1+\sum_{i>0} a_{i} \gamma^{i}=0
$$

taking traces we obtain $\operatorname{Tr}(1)=\varphi\left(2^{e}\right) / 2=0(\bmod 5)$ which is impossible.

If $q_{1,2}\left(\gamma, \gamma^{\ell}\right)=0$, taking traces we obtain

$$
\operatorname{Tr}(1)+\operatorname{Tr}\left(\gamma^{2+\ell}\right)+\operatorname{Tr}\left(\gamma^{2+3 \ell}\right)=0 \quad(\bmod 5) .
$$

Notice that $\operatorname{Tr}\left(\gamma^{2+\ell}\right) \operatorname{Tr}\left(\gamma^{2+3 \ell}\right)=0(\bmod 5)$. From $(17)$, we get that either $\operatorname{gcd}\left(2^{e}, 2+\ell\right)=$ $2^{e-1}$ or $\operatorname{gcd}\left(2^{e}, 2+3 \ell\right)=2^{e-1}$. In the first case, $2+\ell=2^{e-1}$ and $\gamma^{2-\ell}=-1$. In the second case, $2+3 \ell=2^{e-1}$ and $\gamma^{2+3 \ell}=-1$. Since $\Phi_{1}(x)$ is the unique cyclotomic polynomial dividing

$$
\operatorname{Res}\left(q_{1}(x, w), x^{2} w+1, w\right) \cdot \operatorname{Res}\left(q_{1}(x, w), x^{2} w^{3}+1, w\right)
$$

it follows that $q_{1,2}\left(\gamma, \gamma^{\ell}\right) \neq 0$.

If $q_{1,3}\left(\gamma, \gamma^{\ell}\right)=0$, taking traces we obtain

$$
\operatorname{Tr}(1)-2 \operatorname{Tr}\left(\gamma^{2+\ell}\right)-2 \operatorname{Tr}\left(\gamma^{2+3 \ell}\right)=0 \quad(\bmod 5) .
$$

As above $\operatorname{Tr}\left(\gamma^{2+\ell}\right) \operatorname{Tr}\left(\gamma^{2+3 \ell}\right)=0(\bmod 5)$. Since $\operatorname{Tr}\left(\gamma^{2+h \ell}\right)=\operatorname{Tr}(1) / 2(\bmod 5), h \in\{1,2\}$, is not possible, neither is the equality (18).

Consequently, $\Phi_{n}(x)$ does not divide $q_{1, i}\left(x, x^{\ell}\right)(\bmod 5 \mathbb{Z}[x]), i \in\{1,2,3,4\}$, and thus $\Phi_{n}(x)$ does not divide $q_{1}\left(x, x^{\ell}\right)(\bmod 5 \mathbb{Z}[x])$.

The non divisibility with respect to the other factors $q_{i}\left(x^{2}, x^{\ell}\right), i \in\{2,3,4\}$, can be proved in a similar way. Indeed, for $2 \leqslant i \leqslant 4$, let $P_{i}(x)$ be the polynomials in $\mathbb{Z}[x]$ obtained as in (14) but from the polynomial $q_{i}(z, w)$ instead of $q_{1}(z, w)$. Let us consider

$$
U_{i}(x)=\operatorname{Res}\left(q_{i}(x, w), x \frac{\partial}{\partial x} q_{i}(x, w)+k w \frac{\partial}{\partial w} q_{i}(x, w), w\right) .
$$

Concerning $q_{2}\left(x^{2}, x^{\ell}\right)$ and $q_{3}\left(x^{2}, x^{\ell}\right)$, the polynomials $P_{i}(x)$ are non identically zero modulo $p \mathbb{Z}[x]$, except for $p=2$ with $\ell$ even. Therefore, if $n$ has a factor $p \neq 2$, using 
(13), it turns out that $\Phi_{2 n}(x) \nmid q_{2}\left(x^{2}, x^{\ell}\right)$ and $\Phi_{2 n}(x) \nmid q_{3}\left(x^{2}, x^{\ell}\right)$ When $n=2^{e}$, since the polynomials $U_{2}(x)$ and $U_{3}(x)$ satisfy $U_{i}(x) \neq 0(\bmod 2 \mathbb{Z}[x])$, it turns out that $\Phi_{n}(x) \nmid$ $q_{2}\left(x, x^{k}\right)$ and $\Phi_{n}(x) \nmid q_{3}\left(x, x^{k}\right)$.

Regarding $q_{4}\left(x^{2}, x^{\ell}\right)$, the polynomial $P_{4}(x)$ is non identically zero modulo $p \mathbb{Z}[x]$, except for $p=2$ and $\ell$ even or $p=5$. Moreover, $U_{4}(x) \neq 0(\bmod 2 \mathbb{Z}[x])$. So, we have only to consider the case $n=5^{d}, d \geqslant 1$. In such a case, we can derive that the corresponding polynomial $Q_{4}(x)$ obtained as in $(16)$ from $q_{4}(z, w)$ is not identically zero $(\bmod 5 \mathbb{Z}[x])$, unless $\ell \equiv 4(\bmod 5)$. On the other hand, we have that

$$
q_{4}(z, w)=\prod_{i=1}^{10} q_{4, i}(z, w) \quad(\bmod 5 \mathbb{Z}[z, w]),
$$

where

$$
\begin{aligned}
q_{4,1}(z, w) & =w^{2} z-1 \\
q_{4,2}(z, w) & =\left(w^{2} z+1\right)^{2} \\
q_{4,3}(z, w) & =w^{2} z^{2}-w^{2} z-w z-z+1 \\
q_{4,4}(z, w) & =w^{4} z^{3}-w^{4} z^{2}+w^{3} z^{2}+2 w^{2} z^{2}+2 w^{2} z-2 w z+z-1 \\
q_{4,5}(z, w) & =w^{4} z^{3}-w^{4} z^{2}+2 w^{3} z^{2}+2 w^{2} z^{2}+2 w^{2} z-w z+z-1 \\
q_{4,6}(z, w) & =w^{4} z^{3}-w^{4} z^{2}-w^{3} z^{2}+2 w^{2} z^{2}-2 w^{2} z+w z+z-1 \\
q_{4,7}(z, w) & =w^{4} z^{3}-w^{4} z^{2}+w^{3} z^{2}+2 w^{2} z^{2}-2 w^{2} z-w z+z-1 \\
q_{4,8}(z, w) & =w^{4} z^{3}-w^{4} z^{2}+w^{3} z^{2}-2 w^{2} z^{2}-2 w^{2} z-2 w z+z-1 \\
q_{4,9}(z, w) & =w^{4} z^{3}-w^{4} z^{2}+2 w^{3} z^{2}-2 w^{2} z^{2}-2 w^{2} z-w z+z-1 \\
q_{4,10}(z, w) & =w^{4} z^{4}-2 w^{4} z^{3}+w^{4} z^{2}+w^{3} z^{3}-w^{3} z^{2}+2 w^{2} z^{3}+2 w^{2} z-w z^{2}+w z+z^{2}-2 z+1 .
\end{aligned}
$$

Now, by using a similar argument as the one given for $q_{1}(z, w)$ and $n=5^{d}$ we obtain that $\ell+1=5^{d}$, which leads us to a contradiction, since the polynomial

$$
q_{4}\left(x^{2},-1 / x\right)=5(-1+x)^{5} x^{2}(1+x)^{5}\left(9+46 x^{2}+9 x^{4}\right)
$$

is never a multiple of $\Phi_{5^{d}}(x)$.

As we have shown in Theorem 6 the cyclotomic conjecture for $k=4$, we can apply Theorem 4 to prove the nonexistence of almost Moore digraph of diameter $k=4$.

\section{References}

[1] E. T. Baskoro, M. Miller and J. Plesník, On the structure of digraphs with order close to the Moore bound, Graphs Combin. 14 (1998) 109-119.

[2] E. T. Baskoro, M. Miller, J. Plesník and Š. Znám, Regular digraphs of diameter 2 and maximum order, Australa. J. Combinatorics 9 (1994) 291-306. 
[3] E. T. Baskoro, M. Miller, J. Širáň and M. Sutton, Complete characterisation of almost Moore digraphs of degree three, J. Graph Theory 482 (2005) 112-126.

[4] W. G. Bridges and S. Toueg, On the impossibility of directed Moore graphs, J. Combin. Theory Ser. B 29 (1980) 339-341.

[5] J. Conde, J. Gimbert, J. González, J. M. Miret and R. Moreno, Nonexistence of almost Moore digraphs of diameter three, Electronic J. Combin. 15 (2008) \#R87.

[6] M. A. Fiol, I. Alegre and J. L. A. Yebra, Line digraphs iterations and the $(d, k)$ problem for directed graphs, Proc. 10th Int. Symp. Comput. Arch. (Stockholm, 1983) $174-177$.

[7] J. Gimbert, On the existence of ( $d, k)$-digraphs, Discrete Math. 197-198 1-3 (1999) 375-391.

[8] J. Gimbert, Enumeration of almost Moore digraphs of diameter two, Discrete Math. 231 (2001) 177-190.

[9] H. W. Lenstra Jr. and B. Poonen, Personal communication.

[10] M. Miller, J. Gimbert, J. Širáň and Slamin, Almost Moore digraphs are diregular, Discrete Math. 218 (2000) 265-270.

[11] M. Miller and I. Fris, Maximum order digraphs for diameter 2 or degree 2, Pullman Volume of Graphs and Matrices, Lect. Notes Pure Appl. Math. 139 (1992) 269-278.

[12] M. Miller and J. Širáň, Moore graphs and beyond: A survey, Electronic J. Combin. (2005), DS14.

[13] T. Nagell, Introduction to Number Theory. John Wiley \& Sons Inc., New York 1951.

[14] B. L. van der Waerden, Algebra, vol. I, translation of the German seventh ed., Springer (2003).

[15] L. C. Washington, Introduction to Cyclotomic Fields, Springer (1997). 